\title{
Fibromuscular Dysplasia/Carotid Web in Angio-CT Imaging: A Rare Cause of Ischemic Stroke
}

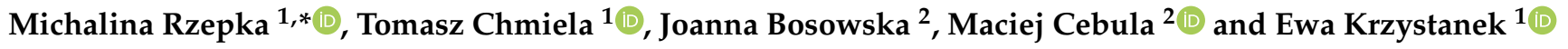 \\ 1 Department of Neurology, Faculty of Medical Sciences in Katowice, Medical University of Silesia, \\ 40-752 Katowice, Poland; tchmiela@sum.edu.pl (T.C.); ekrzystanek@sum.edu.pl (E.K.) \\ 2 Department of Radiodiagnostics, Invasive Radiology and Nuclear Medicine, Department of Radiology and \\ Nuclear Medicine, School of Medicine in Katowice, Medical University of Silesia, 40-752 Katowice, Poland; \\ jbosowska@sum.edu.pl (J.B.); mcebula@sum.edu.pl (M.C.) \\ * Correspondence: michalina.rzepka93@gmail.com
}

Citation: Rzepka, M.; Chmiela, T.; Bosowska, J.; Cebula, M.; Krzystanek, E. Fibromuscular Dysplasia/Carotid Web in Angio-CT Imaging: A Rare Cause of Ischemic Stroke. Medicina 2021, 57, 1112. https://doi.org/ 10.3390/medicina57101112

Academic Editor: Vida Demarin

Received: 2 September 2021

Accepted: 12 October 2021

Published: 15 October 2021

Publisher's Note: MDPI stays neutral with regard to jurisdictional claims in published maps and institutional affiliations.

Copyright: (C) 2021 by the authors. Licensee MDPI, Basel, Switzerland. This article is an open access article distributed under the terms and conditions of the Creative Commons Attribution (CC BY) license (https:/ / creativecommons.org/licenses/by/ $4.0 /)$.
Abstract: Background and Objectives: Carotid web $(\mathrm{CaW})$ is an intimal variant of fibromuscular dysplasia and may constitute as one of rare causes of acute ischemic stroke (AIS). The objective of this study was to determine the prevalence of $\mathrm{CaW}$ in patients with AIS or transient ischemic attack (TIA) based on head/neck CT angiography (CTA) in a Polish cohort study. Materials and Methods: A retrospective study was performed by analyzing 1480 electronic clinical and imaging data regarding patients with AIS or TIA, hospitalized in the years 2018-2020 in the authors' institution. The final sample consisted of 181 patients who underwent head/neck CTA; aged $67.81 \pm 13.51$ years (52\% were women). All head/neck CTA studies were independently evaluated by two radiologists. The patient's clinical condition was assessed with the National Institutes of Health Stroke Scale (NIHSS, $5.76 \pm 4.05$ and $2.88 \pm 3.38$ at admission and at discharge, respectively). Results: 27 patients were identified with CaW. The prevalence of $\mathrm{CaW}$ in the final sample (181 pts with good quality CTA) was $14.9 \%$. In the CaW group, $89 \%$ patients had AIS, including $26 \%$ diagnosed with recurrent and $11 \%$ with cryptogenic strokes. There were no significant differences between the presence of CaW and gender, age, NIHSS score, recurrent or cryptogenic stroke. Conclusions: Our study demonstrated that $\mathrm{CaW}$ may be an underrecognized entity leading to cerebrovascular events. The diagnosis of CaW depends on a high level of awareness and a comprehensive analysis of the neuroimaging studies. Our findings support the hypothesis that it is worthwhile to perform CTA to determine the etiology of ischemic stroke, particularly if predicting factors were not identified.

Keywords: CT angiography; carotid web; ischemic stroke; angiography; fibromuscular dysplasia

\section{Introduction}

Cerebrovascular diseases are one of the major public health problems in industrialized countries. Stroke is a leading cause of permanent disability among adults and the third leading cause of death in Poland, affecting nearly 80,000 individuals annually [1,2]. Acute ischemic stroke (AIS) is defined as a sudden onset of neurologic deficit caused by focal brain ischemia with imaging evidence of acute infarction lasting more than $24 \mathrm{~h}$ (unless interrupted by death or treatment) [3].

An ischemic episode with neurologic deficits but without acute infarction defines transient ischemic attack (TIA) [3]. Up to $80 \%$ of strokes are attributed to brain ischemia, caused by the pathology of large vessel atherosclerosis, small vessel disease, cardiovascular embolism or other rare causes. About 30\% cases of strokes are cryptogenic, in which the cause cannot be evidently established [4].

One rare vessel disease that can be recognized as a cause of stroke is fibromuscular dysplasia (FMD). It is an idiopathic, noninflammatory, nonatherosclerotic vascular disease [5]. FMD most commonly involves the renal, visceral, and craniocervical arteries. It occurs generally in females, middle-aged, and Caucasians [6]. The prevalence of FMD is not 
well-established in the general population. A study reported that the incidence of carotid FMD ranged from $0.9 \%$ to $5.6 \%$ in a population of patients with carotid artery dissection [7]. The most common clinical manifestation of FMD is renovascular hypertension and less frequently neurological symptoms, such as headaches, dizziness, TIA or AIS. FMD can be asymptomatic as well [5].

Carotid web $(\mathrm{CaW})$ is a rare subtype of unifocal FMD and remains as an underrecognized cause of cryptogenic and recurrent ischemic stroke in younger patients without any risk factors [8]. CaW, originally described in 1964 by Palubinskas and Ripley, is defined as a shelf-like defect in a linear filler at the posterior part of the internal carotid artery (ICA) on catheter angiography [9]. Digital subtraction angiography (DSA) is a gold standard for diagnosis of FMD, but current diagnostics generally rely on computed tomography angiography (CTA) [10].

Thus far, it appears that the occurrence of FMD in the carotid arteries has been underestimated in the population of Polish patients with AIS or TIA. In this perspective, we aim to assess the prevalence of $\mathrm{CaW}$ among patients with AIS or TIA based on the head and neck CTA and to characterize the clinical features of patients with CaW.

\section{Materials and Methods}

We performed a retrospective analysis of all patients with AIS or TIA admitted to Central Clinical Hospital of the Medical University of Silesia in Katowice from January 2018 to December 2020. The initial group consisted of 1480 individuals. In the first stage, 1092 patients (pts) without both head and neck CTA were excluded. Then, in a 380 pts study group, 199 pts met the exclusion criteria, which included the conditions preventing a reliable assessment, such as massive atherosclerotic plaques $(n=92)$, lack of carotid CTA $(n=63)$, occlusion of common carotid artery (CCA) and / or ICA $(n=30)$, movement artifacts $(n=9)$ and ICA stent $(n=5)$.

In the final group of $181 \mathrm{pts}$, clinical data regarding gender, age, National Institutes of Health Stroke Scale (NIHSS) score [11], Modified Rankin Scale (mRS) score [12], the stroke side, treatment, bloodwork results and risk factors were collected. Those pts underwent carotid Doppler ultrasound examination. Patients were evaluated for cryptogenic stroke in accordance to criteria of Trial of ORG 10172 in Acute Stroke Treatment (TOAST) [4].

The next step was composed of a blinded reevaluation of patient carotid CTA examinations to find CaW. As there are no formal radiological diagnostic criteria, "string-of-beads", "web-like" defects and focal/tubular lesions other than plaques were classified as CaW [13]. It was performed by two radiologists with 7 and 3 years' experience in CTA evaluation on licensed diagnostic workstations (Advantage Workstation (AW) 4.4 Software, GE Healthcare, Waukesha, WI, USA). Exclusion criteria included conditions preventing a reliable assessment, such as massive atherosclerotic plaques $(n=92)$, lack of carotid CTA $(n=63)$, occlusion of common carotid artery (CCA) and/or ICA $(n=30)$, movement artifacts $(n=9)$ and ICA stent $(n=5)$.

The study group consisted of 181 patients; 94 females (52\%) and 87 males (48\%), aged $67.81 \pm 13.51$ years $(71.99 \pm 13.30$ years and $63.30 \pm 12.29$ years, respectively) (mean $\pm S D$ ). The process of the final group creation is presented in Figure 1.

The statistical analysis was performed with Statistica 13.3 (TIBCO Software Inc. (2017) Statistica (data analysis software system, version 13. http://statistica.io)). The quantitative variables are presented as an arithmetic mean and a standard deviation (normally distributed variable) or a median and the interquartile range (variables of not normal/skewed distribution). The normality of distribution was assessed with the Shapiro-Wilk test. Qualitative variables are presented as absolute values and percentage. 


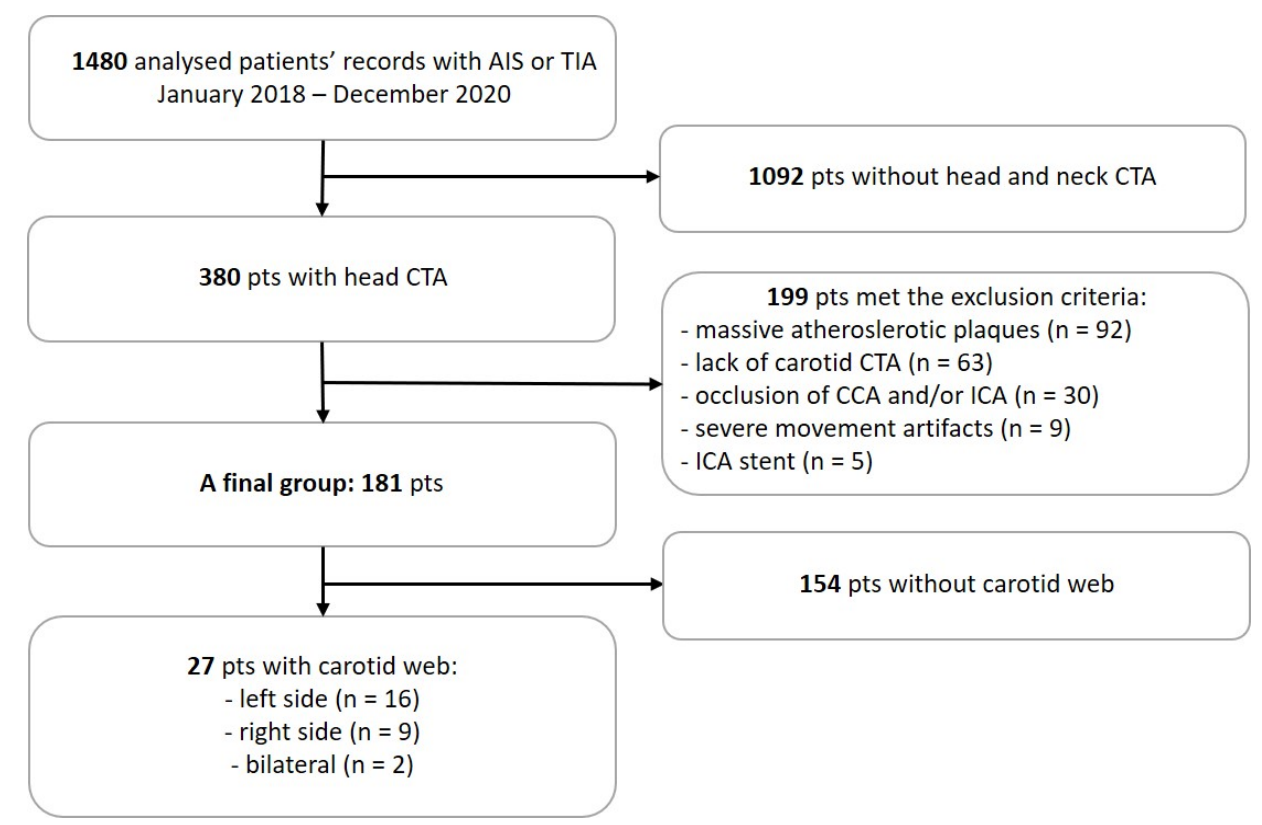

Figure 1. A final group creation process to detect carotid web in patients with AIS or TIA. AIS-acute ischemic stroke; TIA—transient ischemic attack; pts—patients; CTA—computed tomography angiography; $\mathrm{CCA}$ - common carotid artery; and ICA—internal carotid artery.

The intergroup differences for the quantitative variable were assessed with an analysis of variance (normally distributed variables) or the U-Mann-Whitney or the Kruskal-Wallis test (variables of skewed distribution). In the case of statistically significant differences within many groups revealed by the Kruskal-Wallis test, a post-hoc type analysis was performed. Fisher's exact test or chi-square test were performed for qualitative variables. Relationships of quantitative variables were assessed with the Spearman's rank correlation coefficient. Statistical significance was established at $p<0.05$.

Due to the retrospective character of the work and data anonymization, the Ethics Committee of Medical University of Silesia waived the requirement to obtain ethical approval for this study.

\section{Results}

\subsection{The Prevalence of $\mathrm{CaW}$}

In the study group, we found $27 \mathrm{CaWs}$ incuding $16 / 27 \mathrm{CaWs}$ on the left side and 9/27 CaWs on the right side; $2 / 27 \mathrm{CaWs}$ were bilateral. The prevalence of $\mathrm{CaW}$ was $1.8 \%$ in the overall examined cohort, whereas, in the study group, in which we were able to detect $\mathrm{CaW}$, the prevalence of $\mathrm{CaW}$ was $14.9 \%$.

In the CaW group $89 \%$ patients were diagnosed with AIS. We found $10 / 20$ of strokes on the left side, 10/20 were on the right side, and 5/20 patients had CaW on the same side as the cerebral infarction. The CaW side was close to significance with the side of the ischemic stroke ( $p=0.058)$; however, due to the low number of cases in each of the analyzed subgroups, this should not be considered credible. We found that $25 \%(5 / 20)$ stroke patients had $\mathrm{CaW}$ on the ipsilateral side as the cerebral infarction. Among them, three patients had cryptogenic stroke, which may indicate a potential etiology of $\mathrm{CaW}$ as a risk factor. The complete characteristic of final study group is shown in Table 1. 
Table 1. Demographic, clinical and laboratory characteristic of a final analyzed post-stroke and TIA group.

\begin{tabular}{|c|c|c|c|}
\hline \multicolumn{4}{|c|}{ Study Group $(n=181)$} \\
\hline Variable & $\mathrm{CaW}(n=27)$ & no-CaW $(n=154)$ & $p$ Value \\
\hline Gender $[n(\%)]$ & & & 0.39 \\
\hline Females & $16(59 \%)$ & $78(51 \%)$ & \\
\hline Males & $11(41 \%)$ & $76(49 \%)$ & \\
\hline Age [years] [n土SD] & $66.70 \pm 14.34$ & $68.01 \pm 13.40$ & 0.73 \\
\hline \multicolumn{4}{|l|}{ Pathology $[n(\%)]$} \\
\hline Transient ischemic attack & $3(11 \%)$ & $17(11 \%)$ & - \\
\hline Acute supratentorial ischemic stroke & $20(74 \%)$ & $122(79 \%)$ & - \\
\hline Acute infratentorial ischemic stroke & $4(15 \%)$ & $15(10 \%)$ & - \\
\hline NIHSS—admission $[n \pm \mathrm{SD}]$ & $5.76 \pm 4.05$ & $7.51 \pm 5.83$ & 0.27 \\
\hline NIHSS-discharge $[n \pm$ SD] & $2.88 \pm 3.38$ & $5.14 \pm 6.14$ & 0.13 \\
\hline $\operatorname{rtPA}[n(\%)]$ & $17(63 \%)$ & $74(48 \%)$ & 0.12 \\
\hline Thrombectomy $[n(\%)]$ & $3(11 \%)$ & $19(12 \%)$ & 0.94 \\
\hline Stroke side (supratentorial) $[n(\%)]^{1,2}$ & & & 0.89 \\
\hline Right & $10(37 \%)$ & $59(38 \%)$ & \\
\hline Left & $10(37 \%)$ & $63(41 \%)$ & \\
\hline Recurrent stroke [n (\%)] & $7(26 \%)$ & $28(18 \%)$ & 0.38 \\
\hline Cryptogenic stroke $[n(\%)]$ & $3(11 \%)$ & $20(13 \%)$ & 0.99 \\
\hline \multicolumn{4}{|l|}{ Risk factors $[n(\%)]$} \\
\hline Hypertension & $23(85 \%)$ & $129(84 \%)$ & 0.97 \\
\hline Dyslipidemia & $14(52 \%)$ & $80(52 \%)$ & 0.71 \\
\hline Atrial fibrillation & $5(19 \%)$ & $25(16 \%)$ & 0.64 \\
\hline Diabetes mellitus & $4(15 \%)$ & $48(31 \%)$ & 0.12 \\
\hline Smoking & $5(19 \%)$ & $33(21 \%)$ & 0.87 \\
\hline Myocardial infarction & $3(11 \%)$ & $27(18 \%)$ & 0.49 \\
\hline Ischemic heart disease & $8(30 \%)$ & $62(40 \%)$ & 0.43 \\
\hline Arterial narrowing & $8(30 \%)$ & $32(21 \%)$ & 0.22 \\
\hline \multicolumn{4}{|l|}{ Bloodwork results $[n \pm \mathrm{SD}]$} \\
\hline LDL cholesterol [mg/dL] & $130.08 \pm 46.49$ & $112.97 \pm 49.38$ & 0.06 \\
\hline HDL cholesterol [mg/dL] & $52.96 \pm 12.43$ & $47.64 \pm 15.60$ & 0.02 \\
\hline Total cholesterol [mg/dL] & $209.04 \pm 50.33$ & $183.65 \pm 57.36$ & 0.02 \\
\hline Triglycerides [mg/dL] & $125.59 \pm 65.05$ & $124.88 \pm 59.53$ & 0.65 \\
\hline $\mathrm{Hb} \mathrm{A} 1 \mathrm{c}[\%]$ & $6.50 \pm 0.43$ & $7.27 \pm 1.99$ & - \\
\hline
\end{tabular}

SD-Standard Deviation, TIA-Transient Ischemic Stroke, NIHSS-National Institutes of Health Stroke Scale, rtPA—recombinant tissue plasminogen activator, LDL-Low-density lipoprotein, HDL-High-density lipoprotein, HbA1c-hemoglobin A1c, ${ }^{1}$ - two cases of bilateral $\mathrm{CaW}$ were not included in the table, and ${ }^{2}-19$ cases of infratentorial stroke were not included in the Table. U-Mann-Whitney test for quantitative variables and Fisher's exact test for qualitative variables.

Males were significant younger than females and had better outcomes (lower mRS score). Atrial fibrillation was more frequent in women than in men. (Table 2.)

Table 2. Baseline characteristics of men and women in the final group of patients with AIS/TIA.

\begin{tabular}{|c|c|c|c|}
\hline \multirow[b]{2}{*}{ Variable } & \multicolumn{2}{|c|}{ Study Final Group ( $n=181)$} & \multirow[b]{2}{*}{$p *$ Value } \\
\hline & Females $n=94(52 \%)$ & Males $n=87(48 \%)$ & \\
\hline Age [years] $[n \pm \mathrm{SD}]$ & $72.00 \pm 13.30$ & $63.30 \pm 12.29$ & $<0.01$ \\
\hline \multicolumn{4}{|l|}{ Pathology $[n(\%)]$} \\
\hline Transient Ischemic Attack & $12(13 \%)$ & $8(9 \%)$ & 0.24 \\
\hline Acute supratentorial ischemic stroke & $74(79 \%)$ & $68(78 \%)$ & 0.96 \\
\hline Acute infratentorial ischemic stroke & $8(8 \%)$ & $11(13 \%)$ & 0.39 \\
\hline NIHSS—admission $[n \pm$ SD] & $8.04 \pm 6.32$ & $6.38 \pm 4.64$ & 0.09 \\
\hline NIHSS-discharge $[n \pm$ SD] & $5.77 \pm 6.75$ & $3.77 \pm 4.54$ & 0.09 \\
\hline $\operatorname{mRS}[n \pm \mathrm{SD}]$ & $2.59 \pm 2.05$ & $1.86 \pm 1.80$ & 0.02 \\
\hline $\operatorname{rtPA}[n(\%)]$ & $48(51 \%)$ & $43(49 \%)$ & 0.54 \\
\hline Thrombectomy $[n(\%)]$ & $12(13 \%)$ & $10(12 \%)$ & 0.69 \\
\hline
\end{tabular}


Table 2. Cont.

\begin{tabular}{|c|c|c|c|}
\hline \multirow[b]{2}{*}{ Variable } & \multicolumn{2}{|c|}{ Study Final Group $(n=181)$} & \multirow[b]{2}{*}{$p *$ Value } \\
\hline & Females $n=94(52 \%)$ & Males $n=87(48 \%)$ & \\
\hline Supratentorial stroke site $[n(\%)]$ & & & 0.10 \\
\hline Right & $31(33 \%)$ & $38(44 \%)$ & \\
\hline Left & $43(46 \%)$ & $30(35 \%)$ & \\
\hline Recurrent stroke [n (\%)] & $18(19 \%)$ & $17(20 \%)$ & 0.93 \\
\hline Cryptogenic stroke $[n(\%)]$ & $9(10 \%)$ & $14(16 \%)$ & 0.23 \\
\hline \multicolumn{4}{|l|}{ Risk factors $[n(\%)]$} \\
\hline Hypertension & $79(84 \%)$ & $73(84 \%)$ & 0.98 \\
\hline Dyslipidemia & $49(52 \%)$ & $45(52 \%)$ & 0.96 \\
\hline Atrial fibrillation & $24(26 \%)$ & $6(7 \%)$ & $<0.01 *$ \\
\hline Diabetes mellitus & $26(28 \%)$ & $26(30 \%)$ & 0.74 \\
\hline Smoking & $14(15 \%)$ & $24(28 \%)$ & 0.04 \\
\hline Myocardial infarction & $13(14 \%)$ & $17(20 \%)$ & 0.30 \\
\hline Ischemic heart disease & $38(40 \%)$ & $32(37 \%)$ & 0.62 \\
\hline Arterial narrowing & $17(18 \%)$ & $23(26 \%)$ & 0.18 \\
\hline \multicolumn{4}{|l|}{ Bloodwork results [ $n \pm \mathrm{SD}]$} \\
\hline LDL cholesterol [mg/dL] & $120.09 \pm 51.67$ & $110.86 \pm 46.20$ & 0.29 \\
\hline HDL cholesterol [mg/dL] & $51.15 \pm 12.30$ & $45.51 \pm 17.52$ & $<0.01$ \\
\hline Total cholesterol [mg/dL] & $196.08 \pm 58.36$ & $178.46 \pm 54.17$ & 0.05 \\
\hline Triglycerides [mg/dL] & $120.28 \pm 59.71$ & $130.17 \pm 60.75$ & 0.26 \\
\hline $\mathrm{Hb} \mathrm{A} 1 \mathrm{c}[\%]$ & $7.40 \pm 2.05$ & $6.6 \pm 1.14$ & - \\
\hline
\end{tabular}

SD-Standard Deviation, AIS-Acute Ischemic Stroke, TIA-Transient Ischemic Attack, NIHSS-National Institutes of Health Stroke Scale, mRS—-modified Rankin Scale, rtPA—recombinant tissue plasminogen activator, LDL-Low-density lipoprotein, HDL-High-density lipoprotein, $\mathrm{HbA1c}$ - hemoglobin A1c, and * -result with lower reliability due to few number of cases in the subgroup. U-Mann-Whitney test for quantitative variables and Fisher's exact test for qualitative variables.

\subsection{CaW and Non-CaW Groups}

The occurrence of CaW was associated with a higher level of total cholesterol and high-density lipoprotein cholesterol. There were no significant differences between patients with and without $\mathrm{CaW}$ as we considered gender, age, NIHSS score at admission and at discharge or mRS score. We found no significant differences between patients treated with recombinant tissue plasminogen activator (rtPA) or thrombectomy.

\subsection{CaW and Cryptogenic and Recurrent Stroke}

In the $\mathrm{CaW}$ group, there were $7 / 27(26 \%)$ recurrent strokes and 3/27 (11\%) cryptogenic strokes. No significant differences in $\mathrm{CaW}$ patients with cryptogenic or recurrent strokes were observed.

\subsection{CaW and Risk Factors}

There were no significant differences in cardiovascular risk factors, such as hypertension, dyslipidemia, atrial fibrillation, diabetes mellitus, previous myocardial infarction, ischemic heart disease, smoking and arterial narrowing between the groups with $\mathrm{CaW}$ and without CaW.

\subsection{AIS and TIA Groups}

The analysis of AIS and TIA groups showed no significant differences in the occurrence of CaW.

\section{Discussion}

To our knowledge, this is the first study to extensively assess the occurrence of $\mathrm{CaW}$ in cohort of Polish patients with acute cerebrovascular events (AIS/TIA), retrospectively. Our study showed that the diagnosis of CaW was highly underestimated as we considered the causes of ischemic strokes. It corresponds to Wojcik's reports, which clearly show that 
familiarity with this clinical problem varies significantly between different specializations; however, generally, the knowledge about this pathology is low [14].

Our study suggests that CTA were not evaluated in detail for the occurrence of $\mathrm{CaW}$ routinely at admission as CaWs are usually not associated with significant stenosis [8]. This result highlights that little is known about the CaW/FMD as a rare cause of ischemic stroke. By the reevaluation of CTA, we found 27 patients with CaW. Our retrospective study showed the prevalence of $\mathrm{CaW}$ was $1.8 \%$ in the overall cohort (all admitted patients with acute cerebrovascular events), although not every patient had CTA performed.

This result is in the line with other studies of Mei et al., which reported the prevalence of $\mathrm{CaW}$ of $1.6 \%$ in US cohort of patients with ischemic stroke (IS) [15] and 2.2\% in Asian cohort with IS [16]. However, our final study group, which consisted of the patients who met the rigorous inclusion criteria, was much numerous (14.9\%). Likely, such high results were achieved because the CTA scans were retrospectively reevaluated by radiologists.

$\mathrm{CaW}$ appears to have diagnostic challenges because it may not be detected by routine neuroimaging techniques. In recent years, imaging methods have developed, and now we can diagnose craniocervical vascular pathology with a variety of imaging methods. Catheter digital subtraction angiography (DSA) remains the gold standard method to detect and accurately assess $\mathrm{CaW}$ due to the best spatial and contrast resolution; however, because of its invasiveness and high exposure to ionizing radiation, it is less useful in daily routines [17]. Moreover, the range of interest (ROI) during the DSA study is limited to intracranial arteries in order to have as little impact on a person as possible/radiological protection of the patient. Due to the fact that bifurcation of the common carotid artery is often outside a scan area, we cannot assess CaW in routinely performed DSA.

It seems reasonable to use this invasive imaging method for final verification in uncertain cases. As we consider non-invasive methods, our patients had ultrasound (US) Doppler examination of carotid arteries, and we found out patients that were detected with CaW in this study. US is a low-sensitivity method, strongly dependent on the skills and experience of the operator. Therefore, it is not widely used in clinical practice to detect $\mathrm{CaW}$ [14]; however, in selected cases, it may be a sufficient method [18].

The most useful method in the diagnosis of $\mathrm{CaW}$ in daily practice appears to be $\mathrm{CT}$ angiography (CTA) [8]. The examination is well tolerated by patients, widely available, associated with a low risk of complications and is routinely performed in the acute phase of ischemic stroke $[19,20]$. According to some reports, its sensitivity does not differ significantly from that of the DSA examination [21]. CTA should be considered as the first-choice examination, and its current widespread use is confirmed by Wojcik's reports [14].

Our study found no statistically significant differences in demographics, stroke severity, or vascular risk factors between the groups with $\mathrm{CaW}$ and without $\mathrm{CaW}$. Mei et al. also did not find any differences regarding atrial fibrillation and coronary heart disease between those patients [15]. The higher levels of LDL cholesterol and total cholesterol in the patients with $\mathrm{CaW}$ seem to be clinically insignificant.

Other studies confirmed that TIA/AIS can be more common in CaW patients, even with fewer risk factors [22,23]. However, usually the number of patients with $\mathrm{CaW}$ was small, so it was very difficult to determine the predicting factors [23,24]. CaWs pose a serious risk of ischemic stroke, probably due to thrombus development associated with turbulences with the blood flow and stagnation [8]. The optimal management strategy to prevent AIS in patients with CaW is still under investigation. Antiplatelet therapy, carotid angioplasty, invasive treatment (carotidangioplasty, stenting, mechanical thrombectomy or endarterectomy) are the therapeutic options that can be considered. In our study, an antiplatelet therapy (monotherapy) was administered in the majority of patients as the secondary stroke prevention.

Despite this, some patients had a recurrent stroke, which demonstrates that it could be an insufficient prevention. Studies comparing different pharmacotherapy regimens are needed to determine the optimal pharmacological prevention. However, surgical intervention is supposed to be a dedicated method of treatment of $\mathrm{CaW}$ [25]. Haussen et al. 
reported the recurrence rate of $29 \%$ in patients treated with pharmacotherapy, whereas no recurrent stroke/TIA was observed in stented patients [22]. If CaW is found, looking for FMD in other locations should be considered [26].

Our study has limitations. First of all, the diagnosis of $\mathrm{CaW}$ in our final group was based on CTA without DSA or histopathological confirmation. Secondly, because of the retrospective nature of single-center study, selection and sampling bias was possible. The final study group accounted for $12.23 \%$ of the entire group $(n=181 / 1480)$. It is notable that our finding can be underestimated due to the fact that CTA was not performed in the majority of patients with AIS/TIA. In the final group of patients with CTA, the occurrence of CaW may be underestimated due to exclusion criteria.

\section{Conclusions}

Our study demonstrated that $\mathrm{CaW}$ may be an underrecognized entity leading to cerebrovascular events. The diagnosis of $\mathrm{CaW}$ depends on a high level of awareness and a comprehensive analysis of the neuroimaging studies. Our findings support the hypothesis that it worthwhile to perform CTA to find the etiology of ischemic stroke particularly if predicting factors were not identified.

Author Contributions: Conceptualization: M.R., T.C., M.C. and E.K.; methodology, M.R., T.C., J.B., M.C., E.K.; software, M.C.; validation, M.R., T.C., J.B., M.C. and E.K.; formal analysis, M.C., E.K.; investigation, M.R., T.C., J.B., M.C. and E.K. resources, M.R., T.C., J.B., M.C. and E.K.; data curation, M.R., T.C., J.B., M.C. and E.K.; writing-original draft preparation, M.R., T.C., J.B., M.C. and E.K.; writing—review and editing, E.K.; visualization, M.R., T.C., M.C., E.K.; supervision, E.K.; project administration, M.R., E.K. All authors have read and agreed to the published version of the manuscript.

Funding: This research received no external funding.

Institutional Review Board Statement: The study was conducted according to the guidelines of the Declaration of Helsinki. Ethical review and approval were waived for this study, due to the retrospective character of the work and data anonymization. The Ethics Committee of the Medical University of Silesia waived the requirement to obtain the ethical approval for this study.

Informed Consent Statement: Patient consent was waived due to the retrospective character of the work and data anonymization.

Data Availability Statement: The data presented in this study are available on request from the corresponding author.

Conflicts of Interest: The authors declare no conflict of interest.

\section{References}

1. Błażejewska-Hyżorek, B.; Czernuszenko, A.; Członkowska, A.; Ferens, A.; Gąsecki, D.; Kaczorowski, R.; Karaszewski, B.; Karliński, M.; Kaźmierski, R.; Kłysz, B.; et al. Wytyczne postępowania w udarze mózgu. Pol. Przeglad Neurol. 2019, 15 , 1-156. [CrossRef]

2. Kim, J.; Thayabaranathan, T.; Donnan, G.A.; Howard, G.; Howard, V.J.; Rothwell, P.M.; Feigin, V.; Norrving, B.; Owolabi, M.; Pandian, J.; et al. Global Stroke Statistics 2019. Int. J. Stroke 2020, 15, 819-838. [CrossRef] [PubMed]

3. Sacco, R.L.; Kasner, S.E.; Broderick, J.P.; Caplan, L.R.; Connors, J.J.; Culebras, A.; Elkind, M.S.V.; George, M.G.; Hamdan, A.D.; Higashida, R.T.; et al. An updated definition of stroke for the 21st century: A statement for healthcare professionals from the American heart association/American stroke association. Stroke 2013, 44, 2064-2089. [CrossRef] [PubMed]

4. Adams, H.P.; Woolson, R.F.; Biller, J.; Clarke, W. Studies of org 10172 in patients with acute ischemic stroke. Pathophysiol. Haemost. Thromb. 1992, 22, 99-103. [CrossRef] [PubMed]

5. Ossowska, A.; Chwojnicki, K.; Nyka, W.M. Dysplazja włóknisto-mięśniowa-Rzadka przyczyna udaru mózgu. Varia Med. 2019, 3, 363-366.

6. Pappaccogli, M.; Di Monaco, S.; Warchoł-Celińska, E.; Lorthioir, A.; Amar, L.; Aparicio, L.S.; Beauloye, C.; Bruno, R.M.; Chenu, P.; de Leeuw, P.; et al. The European/International Fibromuscular Dysplasia Registry and Initiative (FEIRI)—clinical phenotypes and their predictors based on a cohort of 1000 patients. Cardiovasc. Res. 2021, 117, 950-959. [CrossRef]

7. Shah, K.P.; Peruri, A.; Kanneganti, M.; Gorsch, L.; Ramcharitar, R.; Williams, C.; Clouse, D.; Thomas, M.; Norton, P.T.; Hagspiel, K.D.; et al. Fibromuscular dysplasia: A comprehensive review on evaluation and management and role for multidisciplinary comprehensive care and patient input model. Semin. Vasc. Surg. 2021, 34, 89-96. [CrossRef] 
8. Choi, P.M.C.; Singh, D.; Trivedi, A.; Qazi, E.; George, D.; Wong, J.; Demchuk, A.M.; Goyal, M.; Hill, M.D.; Menon, B.K. Carotid webs and recurrent ischemic strokes in the Era of CT angiography. Am. J. Neuroradiol. 2015, 36, 2134-2139. [CrossRef]

9. Palubinskas, A.J.; Ripley, H.R. Fibromuscular Hyperplasia in Extrarenal Arteries. Radiology 1964, 82, 451-455. [CrossRef]

10. Kim, S.J.; Nogueira, R.G.; Haussen, D.C. Current Understanding and Gaps in Research of Carotid Webs in Ischemic Strokes: A Review. JAMA Neurol. 2019, 76, 355-361. [CrossRef]

11. Brott, T.; Adams, H.P.; Olinger, C.P.; Marle, J.R.; Barsan, W.G.; Biller, J.; Spilker, J.; Holleran, R.; Eberle, R.; Hertzberg, V.; et al. Measurements of acute cerebral infarction: A clinical examination scale. Stroke 1989, 20, 864-870. [CrossRef]

12. Quinn, T.J.; Dawson, J.; Walters, M.R.; Lees, K.R. Reliability of the modified rankin scale: A systematic review. Stroke 2009, 40, 3393-3395. [CrossRef]

13. Varennes, L.; Tahon, F.; Kastler, A.; Grand, S.; Thony, F.; Baguet, J.P.; Detante, O.; Touzé, E.; Krainik, A. Fibromuscular dysplasia: What the radiologist should know: A pictorial review. Insights Imaging 2015, 6, 295-307. [CrossRef]

14. Wojcik, K.; Milburn, J.; Vidal, G.; Tarsia, J.; Steven, A. Survey of current management practices for carotid webs. Ochsner J. 2019, 19, 296-302. [CrossRef]

15. Mei, J.; Chen, D.; Esenwa, C.; Gold, M.; Burns, J.; Zampolin, R.; Slasky, S.E. Carotid web prevalence in a large hospital-based cohort and its association with ischemic stroke. Clin. Anat. 2021, 34, 867-871. [CrossRef] [PubMed]

16. Yu, Y.; Wang, B.; Zheng, S.; Kou, J.; Gu, X.; Liu, T. Carotid web and ischemic stroke: A CT angiography study. Clin. Imaging 2020, 67, 86-90. [CrossRef] [PubMed]

17. Olindo, S.; Marnat, G.; Chausson, N.; Turpinat, C.; Smadja, D.; Gaillard, N. Carotid webs associated with ischemic stroke. Updated general review and research directions. Rev. Neurol. 2021, 177, 627-638. [CrossRef]

18. Fu, W.; Crockett, A.; Low, G.; Patel, V. Internal carotid artery web: Doppler ultrasound with CT angiography correlation. J. Radiol. Case Rep. 2015, 9, 1-6. [CrossRef] [PubMed]

19. Berge, E.; Whiteley, W.; Audebert, H.; De Marchis, G.M.; Fonseca, A.C.; Padiglioni, C.; de la Ossa, N.P.; Strbian, D.; Tsivgoulis, G.; Turc, G. European Stroke Organisation (ESO) guidelines on intravenous thrombolysis for acute ischaemic stroke. Eur. Stroke J. 2021, 6, I-LXII. [CrossRef]

20. Mayer, S.A.; Viarasilpa, T.; Panyavachiraporn, N.; Brady, M.; Scozzari, D.; Van Harn, M.; Miller, D.; Katramados, A.; Hefzy, H.; Malik, S.; et al. CTA-for-All: Impact of Emergency Computed Tomographic Angiography for All Patients With Stroke Presenting Within 24 Hours of Onset. Stroke 2020, 51, 331-334. [CrossRef]

21. Madaelil, T.P.; Grossberg, J.A.; Nogueira, R.G.; Anderson, A.; Barreira, C.; Frankel, M.; Haussen, D.C. Multimodality Imaging in Carotid Web. Front. Neurol. 2019, 10, 3-7. [CrossRef] [PubMed]

22. Haussen, D.C.; Grossberg, J.A.; Bouslama, M.; Pradilla, G.; Belagaje, S.; Bianchi, N.; Allen, J.W.; Frankel, M.; Nogueira, R.G. Carotid web (intimal Fibromuscular Dysplasia) has high stroke recurrence risk and is amenable to stenting. Stroke 2017, 48, 3134-3137. [CrossRef] [PubMed]

23. Sajedi, P.; Chelala, L.; Nunez-Gonalez, J.; Cronin, C.; Kittner, S.; Zhuo, J.; Zhang, Y.; Gandhi, D.; Raghavan, P. Carotid webs and ischemic stroke: Experiences in a comprehensive stroke center. J. Neuroradiol. 2019, 46, 136-140. [CrossRef] [PubMed]

24. Compagne, K.C.J.; van Es, A.C.G.M.; Berkhemer, O.A.; Borst, J.; Roos, Y.B.W.E.; van Oostenbrugge, R.J.; van Zwam, W.H.; Majoie, C.B.L.M.; Marquering, H.A.; Dippel, D.W.J.; et al. Prevalence of carotid web in patients with acute intracranial stroke due to intracranial large vessel occlusion. Radiology 2018, 286, 1000-1007. [CrossRef]

25. Phair, J.; Trestman, E.B.; Yean, C.; Lipsitz, E.C. Endarterectomy for a symptomatic carotid web. Vascular 2017, 25, 553-556. [CrossRef]

26. Warchol-Celinska, E.; Prejbisz, A.; Dobrowolski, P.; Klisiewicz, A.; Kadziela, J.; Florczak, E.; Michalowska, I.; Jozwik-Plebanek, K.; Kabat, M.; Kwiatek, P.; et al. Systematic and multidisciplinary evaluation of fibromuscular dysplasia patients reveals high prevalence of previously undetected fibromuscular dysplasia lesions and affects clinical decisions: The ARCADIA-POL Study. Hypertension 2020, 75, 1102-1109. [CrossRef] 